\title{
On Distance in Complements of Graphs
}

\author{
Ivan Gutman, Jia Lu, Mohamed Amine Boutiche
}

\begin{abstract}
Let $G$ be a graph with $n$ vertices and $m$ edges. In many cases the complement of $G$ has the following properties: it is connected, its diameter is 2 , its Wiener index is equal to $\left(\begin{array}{l}n \\ 2\end{array}\right)+m$, and its hyper-Wiener index is equal to $\left(\begin{array}{l}n \\ 2\end{array}\right)+2 m$. We characterize the graphs whose complements have the mentioned properties.
\end{abstract}

Keywords: Distance (in graph), complement (of graph), Wiener index, hyper-Wiener index

\section{Introduction}

In this paper we are concerned with simple graphs, that is graphs without weighted, directed, or multiple edges, and without self loops. Let $G$ be such a graph and let $V(G)$ and $E(G)$ be, respectively, its vertex and edge sets. Let $n=|V(G)|$ and $m=|E(G)|$ be, respectively, the number of vertices and edges of $G$. The edge of $G$, connecting the vertices $x$ and $y$ will be denoted by $x y$.

The complement $\bar{G}$ of $G$ is the graph whose vertex set is $V(G)$, and in which two vertices are adjacent if and only if they are not adjacent in $G$. Thus, $u v \in E(\bar{G})$ if and only if $u v \notin E(G)$, and $|E(\bar{G})|=\left(\begin{array}{l}n \\ 2\end{array}\right)-m$.

Let $u$ and $v$ be two vertices of the graph $G$. The distance $d_{G}(u, v)$ of these vertices is the length of (= number of edges in) a shortest path that connects $u$ and $v$ [1]. If such a path does not exist (which happens if $G$ is not connected), then the distance between $u$ and $v$ is not defined.

If the graph $G$ is connected, then the greatest distance between two of its vertices is the diameter of $G$, denoted by $\operatorname{diam}(G)$.

Manuscript received January 14, 2015; accepted June 1, 2015.

I. Gutman is with the Faculty of Science, University of Kragujevac, Serbia, and the State University of Novi Pazar, Serbia; J. Lu is with the Department of Mathematics, Central South University, Changsha 41075, P. R. China; M. A. Boutiche is with the Faculty of Mathematics, Université des Sciences et de la Technologie Houari Boumediene, El Alia, Bab Ezzouar 16111, Algeria 
If the graph $G$ is connected, then its Wiener index and hyper-Wiener index are defined as

$$
W=W(G)=\sum_{\{u, v\} \subseteq V(G)} d_{G}(u, v)
$$

and

$$
W W=W W(G)=\frac{1}{2} \sum_{\{u, v\} \subseteq V(G)}\left[d_{G}(u, v)+d_{G}(u, v)^{2}\right] .
$$

For details on these much studied structure descriptors see the recent review [2] and the references cited therein.

In a recent paper [3], it was observed that if a graph $G$ has the property $\mathbf{X}$ (defined below), then the Wiener index of its complement depends only on the parameters $n$ and $m$, and is insensitive of any other structural details of the graphs $G$ or $\bar{G}$. We now examine this phenomenon in more detail.

Definition 1 We say that the graph $G$ has property $\mathbf{X}$ if for every edge xy (i.e., pair of adjacent vertices $x$ and $y$ ), there exists a vertex $z$, such that $z$ is not adjacent to either $x$ or $y$.

The significance of property $\mathbf{X}$ is seen from its following (easy) consequences with regard to the complement of the graph $G$.

Let $G$ be a graph with $n$ vertices and $m$ edges. Let $\bar{G}$ be its complement.

Proposition 1 If the graph $G$ has property $\mathbf{X}$, then $\bar{G}$ is connected.

Proof Let $x$ and $y$ be two vertices of the graph $G$. If $x$ and $y$ are not adjacent in $G$, then they are adjacent in $\bar{G}$, and thus $d_{\bar{G}}(x, y)=1$. If $x$ and $y$ are adjacent in $G$, then by property $\mathbf{X}$, there is a vertex $z$ not adjacent to either $x$ or $y$. Then in $\bar{G}, x$ and $z$ are adjacent, $y$ and $z$ are adjacent, and $x$ and $y$ are not adjacent. Consequently, in $\bar{G}$, the vertices $x$ and $y$ are connected by a path $x z y$ of length 2 , i.e., $d_{\bar{G}}(x, y)=2$.

Since $1 \leq d_{\bar{G}}(x, y) \leq 2$ holds for any two vertices of $\bar{G}$, this graph is connected.

The above proof immediately implies:

Proposition 2 If the graph $G$ has property $\mathbf{X}$, then $\operatorname{diam}(\bar{G})=2$.

Proposition 3 If the graph $G$ has property $\mathbf{X}$, then

$$
W(\bar{G})=\left(\begin{array}{l}
n \\
2
\end{array}\right)+m
$$


Proof From the proof of Proposition 1 we known that in $\bar{G}$ there are $m$ pairs of vertices at distance 2. The remaining $\left(\begin{array}{l}n \\ 2\end{array}\right)-m$ pairs of vertices have distance 1. Therefore, by Eq. (1),

$$
W(\bar{G})=\left[\left(\begin{array}{l}
n \\
2
\end{array}\right)-1\right] \times 1+m \times 2
$$

resulting in Eq. (3).

Proposition 4 If the graph $G$ has property $\mathbf{X}$, then

$$
W W(\bar{G})=\left(\begin{array}{l}
n \\
2
\end{array}\right)+2 m .
$$

Proof Repeating the arguments from the proof of Proposition 3, and bearing in mind Eq. (2), we get

$$
W W(\bar{G})=\frac{1}{2}\left(\left[\left(\begin{array}{l}
n \\
2
\end{array}\right)-1\right] \times 1+m \times 2\right)+\frac{1}{2}\left(\left[\left(\begin{array}{l}
n \\
2
\end{array}\right)-1\right] \times 1+m \times 2^{2}\right)
$$

resulting in Eq. (4).

\section{Graphs possessing property $X$}

We start with the obvious:

Theorem 1 If the graph $G$ is disconnected, then $G$ has property $\mathbf{X}$.

If $G$ is connected, then we have:

Theorem 2 If $G$ is a connected graph, and $\operatorname{diam}(G) \geq 4$, then $G$ has property $\mathbf{X}$.

Proof If $\operatorname{diam}(G) \geq 4$, then in $V(G)$ there must exist five vertices, say, $p, q, r, s, t$, such that $p q, q r, r s, s t \in E(G)$, and $d_{G}(p, t)=4$. Because of $d_{G}(p, t)=4$, the vertex $p$ cannot be adjacent to $r, s, t$, the vertex $q$ cannot be adjacent to $s, t$, and the vertex $r$ cannot be adjacent to $t$.

Now, for the edge $p q$, the vertex $s$ satisfies the requirement of Definition 1 . The same is true for the edges $q r, r s, s t$, and the vertices $t, p$, and $q$, respectively.

Let $x$ and $y$ be some other pair of adjacent vertices of $G$. If neither $x$ nor $y$ is adjacent to $p$, then we are done. Let, therefore, $x$ be adjacent to $p$. Then the vertex $x$ cannot be 
adjacent to vertex $t$, because otherwise there would exist a path pxt of length two, which is impossible because of $d_{G}(p, t)=4$. Also the vertex $y$ cannot be adjacent to vertex $t$, because otherwise there would exist a path pxyt of length three, which is impossible because of $d_{G}(p, t)=4$. Thus, either $p$ or $t$ satisfy the requirement of Definition 1 with regard to the edge $x y$.

Therefore, all edges of $G$ satisfy the requirement of Definition 1. Therefore, $G$ has property $\mathbf{X}$.

Theorem 2 shows that graphs possessing property $\mathbf{X}$ abound, and that most graphs encountered in any applications of graph theory possess property $\mathbf{X}$. In additions, there exists graphs with diameter smaller than 4 that also have property $\mathbf{X}$. Some of these are depicted in Fig. 1.
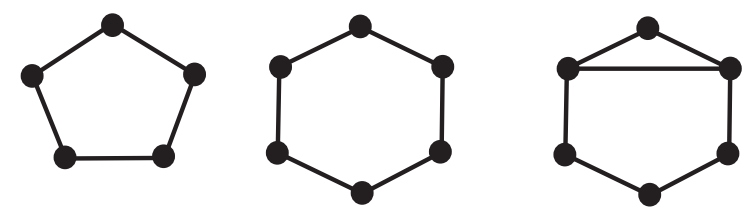

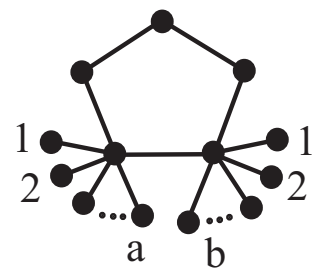

$a, b \geqslant 0$

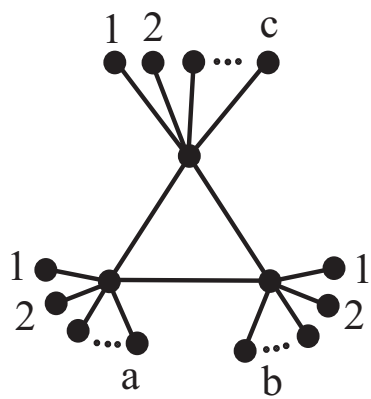

$a, b, c>0$

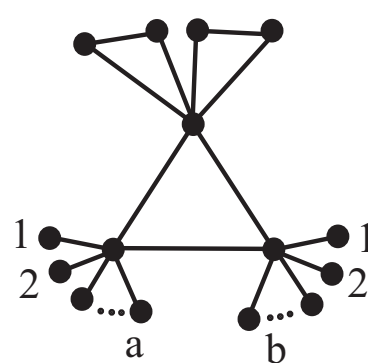

$a, b \geqslant 0$

Fig. 1. Graphs with small diameter having property $\mathbf{X}$. Except the pentagon, all other such graphs have diameter equal to 3 .

\section{Graphs possessing property $Y$}

In view of Theorems 1 and 2, as well as the examples shown in Fig. 1, it appears to be more convenient to consider graphs without property $\mathbf{X}$. These graphs must then have the following property $\mathbf{Y}$. 
Definition 2 We say that the graph $G$ has property $\mathbf{Y}$ if it has at least one edge xy (i.e., a pair of adjacent vertices $x$ and $y$ ), such that all other vertices of $G$ are adjacent either to $x$ or to $y$ or to both $x$ and $y$.

Evidently, any graph $G$ has either property $\mathbf{X}$ or property $\mathbf{Y}$.

According to Definition 2, the general structure of a graph $\mathscr{G}$ with property $\mathbf{Y}$ must be as follows: $\mathscr{G}$ possesses adjacent vertices $x$ and $y$. Its vertex set is partitioned into three parts: $V(\mathscr{G})=A \cup B \cup C$, where $A$ is the set of vertices adjacent to vertex $x, B$ is the set of vertices adjacent to vertex $y$, and $C$ is the set of vertices adjacent to both $x$ and $y$. Let $|A|=a,|B|=b$ and $|C|=c$. Without loss of generality, throughout this paper it will be assumed that $a \geq b$.

The $\mathscr{G}$-type graph with fixed values of the parameters $a, b, c$ and with minimal number of edges has the structure shown in Fig. 2.

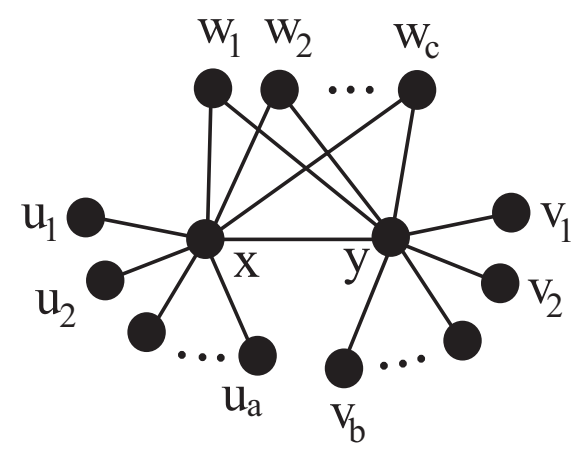

$$
\mathrm{G}(\mathrm{a}, \mathrm{b}, \mathrm{c})
$$

Fig. 2. The graph possessing property $\mathbf{Y}$ with minimal number of edges, for given values of the parameters $a, b, c \geq 0$.

Definition 2 immediately implies the following:

Proposition 5 If a graph $G$ has property $\mathbf{Y}$, then any graph obtained by adding one or more edges to $G$ has property $\mathbf{Y}$.

The cyclomatic number of a connected graph $G$ with $n$ vertices and $m$ edges is $\gamma(G)=$ $m-n+1$ [4]. Connected graphs with $\gamma=1$ are the trees, whereas graphs with $\gamma=1,2,3, \ldots$ are said to be unicyclic, bicyclic, tricyclic, ....

From Fig. 2 it is evident that $\gamma(G(a, b, c))=c$. From this fact, and bearing Proposition 5 in mind, we can now characterize all graphs with property $\mathbf{Y}$ and cyclomatic number $\gamma=0,1,2, \ldots$ 


\section{Trees with property $Y$}

The characterization of trees (connected graphs with cyclomatic number $\gamma=0$ ) with property $\mathbf{Y}$ is elementary [3]. The graph of type $G(a, b, c)$ (cf. Fig. 2) is a tree if $c=0$. Adding more edges to $G(a, b, c)$ (cf. Proposition 5) would increase the cyclomatic number. Therefore, all trees with property $\mathbf{Y}$ are of the type $G(a, b, 0)$. If $a \geq b=0$, then this is the star. If $a \geq b>0$ then this is the double star.

\section{Unicyclic graphs with property $Y$}

There are two classes of graphs with $\gamma=1$, having property $\mathbf{Y}$. The first class consists of $G(a, b, 1)$ whereas the second class consists of $G(a, b, 0)$ to which one new edge is added.

An edge can be added to $G(a, b, 0)$ in two distinct ways. Using the notation specified in Fig. 2, the new edge may be either $u_{1} u_{2}$ or $u_{1} v_{1}$.

The structure of the three types of unicyclic graphs thus constructed is depicted in Fig. 3

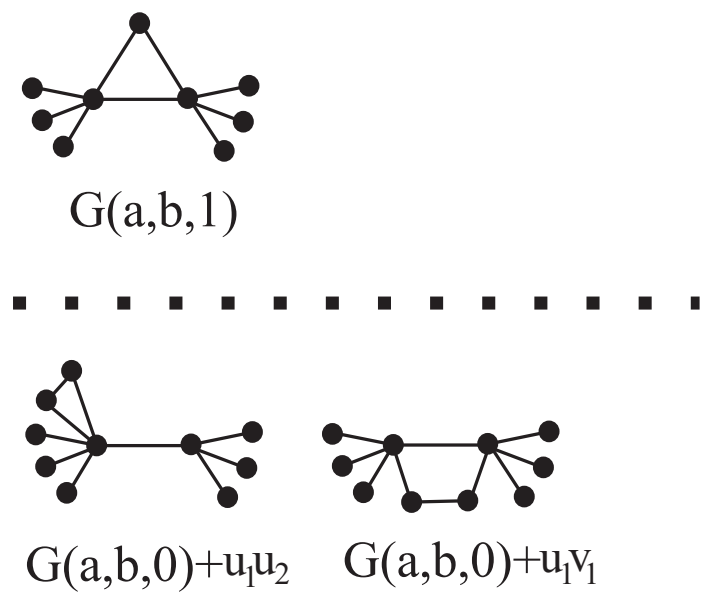

Fig. 3. All possible types of unicyclic graphs possessing property $\mathbf{Y}$. These graphs may have any number of pendent vertices attached to the vertices $x$ and $y$, including the case when there are no pendent vertices attached to $x$ and/or $y$.

\section{Bicyclic graphs with property $Y$}

There are three classes of graphs with $\gamma=2$, having property $\mathbf{Y}$. The first class consists of $G(a, b, 2)$, the second class consists of $G(a, b, 1)$ to which one edge is added, the third class consists of $G(a, b, 0)$ to which two edges are added. 
One edge can be added to $G(a, b, 1)$ in three distinct ways. Using the notation from Fig. 2 , this edge may be either $u_{1} u_{2}$ or $u_{1} v_{1}$ or $u_{1} w_{1}$.

Two edges can be added to $G(a, b, 0)$ in seven distinct way. These are $u_{1} u_{2}+u_{1} u_{3}$, $u_{1} u_{2}+u_{3} u_{4}, u_{1} u_{2}+u_{1} v_{1}, u_{1} u_{2}+u_{3} v_{1}, u_{1} u_{2}+v_{1} v_{2}, u_{1} v_{1}+u_{2} v_{1}, u_{1} v_{1}+u_{2} v_{2}$.

The structure of the eleven $(=1+3+7)$ types of bicyclic graphs thus constructed is depicted in Fig. 4.

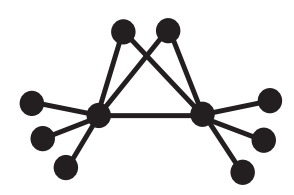

$\mathrm{G}(\mathrm{a}, \mathrm{b}, 2)$

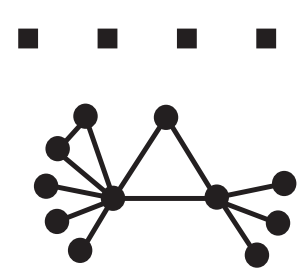

$\mathrm{G}(\mathrm{a}, \mathrm{b}, 1)+\mathrm{u}_{1} \mathrm{u}_{2}$

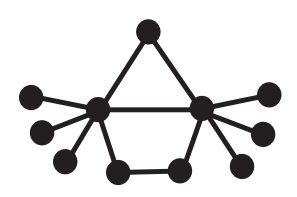

$\mathrm{G}(\mathrm{a}, \mathrm{b}, 1)+\mathrm{u}_{1} \mathrm{v}_{1}$

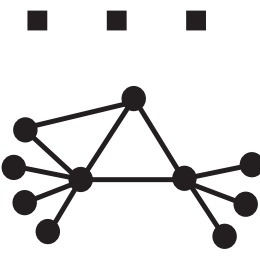

$\mathrm{G}(\mathrm{a}, \mathrm{b}, 1)+\mathrm{u}_{1} \mathrm{w}_{2}$

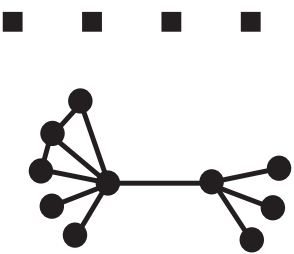

$\mathrm{G}(\mathrm{a}, \mathrm{b}, 0)+\mathrm{u}_{1} \mathrm{u}_{2}+\mathrm{u}_{1} \mathrm{u}_{3}$

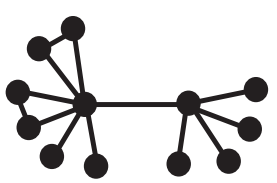

$\mathrm{G}(\mathrm{a}, \mathrm{b}, 0)+\mathrm{u}_{1} \mathrm{u}_{2}+\mathrm{u}_{3} \mathrm{u}_{4}$

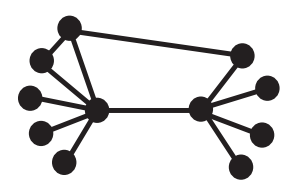

$\mathrm{G}(\mathrm{a}, \mathrm{b}, 0)+\mathrm{u}_{1} \mathrm{u}_{2}+\mathrm{u}_{1} \mathrm{v}_{1}$

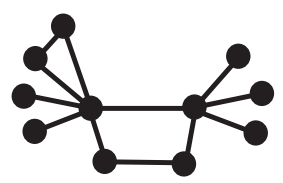

$\mathrm{G}(\mathrm{a}, \mathrm{b}, 0)+\mathrm{u}_{1} \mathrm{u}_{2}+\mathrm{u}_{3} \mathrm{v}_{1}$

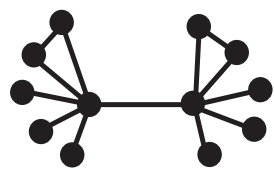

$\mathrm{G}(\mathrm{a}, \mathrm{b}, 0)+\mathrm{u}_{1} \mathrm{u}_{2}+\mathrm{v}_{1} \mathrm{v}_{2}$

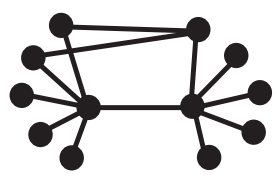

$\mathrm{G}(\mathrm{a}, \mathrm{b}, 0)+\mathrm{u}_{1} \mathrm{v}_{1}+\mathrm{u}_{2} \mathrm{v}_{1}$

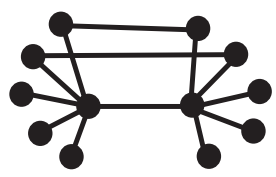

$\mathrm{G}(\mathrm{a}, \mathrm{b}, 0)+\mathrm{u}_{1} \mathrm{v}_{1}+\mathrm{u}_{2} \mathrm{v}_{2}$

Fig. 4. All possible types of bicyclic graphs possessing property $\mathbf{Y}$. Other details are same as in Fig. 3. 


\section{Concluding remarks}

By means of the present analysis, we were able to characterize all graphs with cyclomatic number $\gamma=0,1,2$, that have property $\mathbf{Y}$, i.e., those for which Eqs. (1) and (2) are violated. By this, we have extended and corrected the results of an earlier work [3].

Considerations of the same kind could be continued also for the case $\gamma=3$ (and possibly also for $\gamma>3$ ). However, the number of types of graphs that have to be distinguished becomes prohibitively large and therefore we did not pursue our study beyond $\gamma=2$.

\section{References}

[1] F. Buckley, F. Harary, Distance in Graphs, Addison-Wesley, Redwood, 1990.

[2] K. Xu, M. LiU, K. C. DAs, I. Gutman, B. Furtula, A survey on graphs extremal with respect to distance-based topological indices, MATCH Commun. Math. Comput. Chem., Vol. 71, 3 (2014), 461-508.

[3] J. Senbagamalar, J. Baskar Babujee, I. Gutman, On Wiener index of graph complements, Trans. Comb., Vol. 3, 2 (2014), 11-15.

[4] F. HaRARY, Graph Theory, Addison-Wesley, Reading, 1969. 